\title{
PRESENÇA DE ANEMIA, ADESÃO E TEMPO DE SUPLEMENTAÇÃO COM SULFATO FERROSO EM PRÉ-ESCOLARES DE VENÂNCIO AIRES, RS
}

\author{
Liziane Hermes ${ }^{1}$ \\ Maiara de Queiroz Fischer ${ }^{2}$ \\ Josiane Pereira Pacheco ${ }^{3}$ \\ Luiza Muller ${ }^{4}$ \\ Luana Beatriz Limberger ${ }^{5}$ \\ Caroline dos Santos ${ }^{6}$ \\ Patrícia Molz \\ Karini da Rosa ${ }^{8}$ \\ Daniel Prá ${ }^{9}$ \\ Silvia Isabel Rech Franke ${ }^{10}$
}

\begin{abstract}
RESUMO
A anemia, uma síndrome clínica multifatorial, caracteriza-se pela diminuição na concentração de hemoglobina no sangue em consequência da carência de ferro ou de outros nutrientes essenciais. Este trabalho teve como objetivo reavaliar a presença de anemia nas mesmas crianças que participaram do estudo em 2012, bem como avaliar a taxa de adesão e tempo de suplementação ao programa nacional de suplementação de sulfato ferroso das mesmas crianças avaliadas em 2012 e reavaliadas em 2013. A amostra de 2013 foi composta por 36 crianças, que já haviam participado do estudo em 2012. No grupo reavaliado em 2013, a presença de anemia foi de $8,3 \%$ e a taxa de adesão à suplementação foi de $80 \%$, contudo, o tempo médio de suplementação foi de apenas 4,5 meses. Concluímos que houve adesão inadequada (tempo curto) à suplementação de sulfato ferroso nas crianças. É possível que o baixo percentual de anemia encontrado possa ser justificado pela orientação nutricional dada aos pais após diagnostico de anemia na pesquisa realizada em 2012.
\end{abstract}

Palavras-chave: Anemia. Crianças. Suplementação. Sulfato Ferroso.

\footnotetext{
${ }^{1}$ Mestranda em Promoção da Saúde pela Universidade de Santa Cruz do Sul - UNISC. <lizihermes@yahoo.com.br>

${ }^{2}$ Acadêmica do Curso de Nutrição Universidade de Santa Cruz do Sul - UNISC. <maiarafischer@gmail.com>

${ }^{3}$ Mestre em Promoção da Saúde pela Universidade de Santa Cruz do Sul - UNISC. <josi-pp@ @ol.com.br>

${ }^{4}$ Mestranda em Promoção da Saúde pela Universidade de Santa Cruz do Sul -UNISC. <muller.luiza@yahoo.com.br>

${ }^{5}$ Acadêmica do Curso de Nutrição da Universidade de Santa Cruz do Sul - UNISC. <luanalimberger@mx2.unisc.br>

${ }^{6}$ Acadêmica do Curso de Nutrição da Universidade de Santa Cruz do Sul - UNISC. <carolschaack@hotmail.com>

${ }^{7}$ Mestranda em Promoção da Saúde da Universidade de Santa Cruz do Sul - UNISC. <patrícia.molz@gmail.com>
Mestranda em Promoção da Saúde pela Universidade de Santa Cruz do Sul - UNISC. $<$ karini_drosa@hotmail.com>

${ }^{9}$ Docente Departamento de Biologia e Farmácia e do Programa de Pós-Graduação em Promoção da Saúde na Universidade de Santa Cruz do Sul - UNISC. <dpra@unisc.br>

${ }^{10}$ Docente do Departamento de Educação Física e Saúde e do Programa de Pós-Graduação em Promoção da Saúde da Universidade de Santa Cruz do Sul - UNISC. 〈silviafr@unisc.br〉
} 


\begin{abstract}
Anemia is a multifactorial clinical syndrome characterized by a decrease in hemoglobin concentration in blood as a result of iron deficiency or other essential nutrients. This study aimed to reassess the presence of anemia in the same children whom participated in the study in 2012, also to evaluate the attachment rate and the supplementation time to the national ferrous sulfate program of the same children assessed in 2012 and reassessed in 2013 program. The 2013 sample consisted of 36 children who had participated in the study in 2012. In the revalued group from in 2013, the presence of anemia was $8.3 \%$ and the rate of adherence to supplementation was $80 \%$, however, the average supplementation time was only 4.5 months. We concluded that the adherence was inadequate (short time) to ferrous sulfate supplementation in children. It is possible that the low percentage of anemia found can be explained by the nutritional advice given to parents after diagnosis of anemia in the search conducted in 2012.
\end{abstract}

Keywords: Anemia. Children. Supplementation. Ferrous Sulfate.

\title{
1 INTRODUÇÃO
}

Conhecida como uma síndrome clínica, a anemia caracteriza-se pela diminuição na concentração de hemoglobina no sangue em consequência da carência de ferro ou de outros nutrientes essenciais. Os níveis de hemoglobina variam de acordo com a idade e o sexo (OMS, 2001). A anemia atinge 1,62 bilhões de pessoas, número este que corresponde a $24,8 \%$ da população mundial. A mais alta prevalência (cerca de 50\% em alguns países) ocorre na população com idade pré-escolar e tem como principal causa a deficiência de ferro (AZEREDO et al., 2010; OMS, 2008).

A anemia por deficiência de ferro é o distúrbio nutricional que continua persistindo como um dos mais graves problemas de saúde pública no mundo (MOHAMED et al., 2010; LOZOFF et al., 2006). A anemia atinge principalmente os grupos mais vulneráveis à carência de ferro, que são as crianças e as gestantes, em função do aumento das necessidades desse mineral. A anemia ferropriva é a única carência de nutriente que é significativamente prevalente em praticamente todos os países, mas atinge principalmente os países em desenvolvimento.

Muitos fatores podem influenciar na origem da anemia, como o baixo nível socioeconômico, a prematuridade, o baixo peso de nascimento, a interrupção precoce do aleitamento materno, alimentação inadequada com ingestão precoce de leite de vaca e/ou alimentos sólidos, ingestão frequente e excessiva de chá, baixa ingestão de carne ou de vitamina $\mathrm{C}$, aleitamento materno por mais de 6 meses sem suplementação de ferro, a baixa ingestão de alimentos fonte desse mineral, má absorção de ferro e elevadas necessidades 
fisiológicas em alguns períodos da vida, como períodos de intenso crescimento (NEUMANN et al., 2000; SILVA et al., 2011).

Em sua fase mais avançada, a anemia está associada a sintomas clínicos como fraqueza, diminuição da capacidade respiratória e tontura (PAIVA; RONDÓA; SHINOHARAB, 2000). Pode prejudicar o desenvolvimento mental e psicomotor, causar aumento da morbimortalidade materna e infantil, além de diminuir o desempenho do indivíduo no trabalho e redução da resistência às infecções (JORDÃO; BERNARDI; BARROS, 2009).

A prevenção da anemia deve ser feita com ações de educação nutricional, atenção básica, estratégias sanitárias, acesso a alimentos ricos em ferro e fortificados, incentivo ao aleitamento materno e suplementação profilática (AZEREDO et al., 2010; OMS, 2008). Em 2005, o Ministério da Saúde criou o Programa Nacional de Suplementação de Ferro (PNSF) como estratégia para o controle da anemia no Brasil. Esse programa preconiza a suplementação semanal com sulfato ferroso (25 mg de ferro) para lactentes de 6 a 18 meses de idade. Se a criança não estiver mais em aleitamento materno exclusivo, a suplementação deverá ser feita a partir do $4^{\circ}$ mês até $18^{\circ}$ mês de idade (AZEREDO et al., 2010; BRASIL, 2005). Para o sucesso do PNSF, é imprescindível que as famílias se sensibilizem da importância da suplementação e apresentem adesão à administração do sulfato ferroso para as crianças (AZEREDO et al., 2010; BRASIL, 2005; BORTOLINI; VITOLO, 2007).

Este trabalho teve como objetivo reavaliar a presença de anemia nas mesmas crianças que participaram do estudo em 2012, bem como avaliar o tempo de suplementação e a taxa de adesão ao programa nacional de suplementação de sulfato ferroso das mesmas crianças avaliadas em 2012 e reavaliadas em 2013.

\section{METODOLOGIA}

A amostra foi composta por crianças matriculadas nas escolas da rede municipal de Venâncio Aires, RS, em 2012 e 2013 cujos pais haviam aceitado participar do estudo em 2012, bem como participar do estudo em 2013.

No ano de 2013, foi realizado um estudo transversal pertencente ao projeto intitulado Relação entre concentração sanguínea de ferro, desenvolvimento cognitivo e saúde genômica 
em crianças com idade entre 14 e 38 meses $^{11}$, no qual foi reavaliada a presença de anemia e avaliado o tempo de suplementação e a taxa de adesão ao programa nacional de suplementação de sulfato ferroso das mesmas crianças do estudo de 2012, cujos pais aceitaram participar do estudo (BRASIL, 2005).

O tempo de administração da suplementação de sulfato ferroso da criança foi obtido por meio de questionamento presencial realizado à mãe ou responsável pelo pesquisador principal e entrevistadores treinados. As análises sanguíneas foram realizadas, em dia préagendado com os pais, por profissional habilitado, em um laboratório de análises clínicas do município. Foi realizado hemograma completo, utilizando o analisador automático da marca Symex, modelo XS 1000i. Para diagnóstico de anemia foi utilizado como ponto de corte hemoglobina de < 11,0 g/dL (OMS, 2001).

Para a análise estatística foi utilizado o programa Statistical Package for Social Sciences (SPSS) versão 18.0 (Chicago, IL) e o gráfico elaborado no GraphPad Prism versão 4.0 (San Diego, CA). O teste t de Student pareado foi empregado. O nível de significância utilizado foi de $\mathrm{p}<0,05$.

\section{RESULTADOS E DISCUSSÃO}

Das 113 crianças avaliadas no estudo realizado em 2012 (PACHECO et al., 2013), somente 36 foram reavaliadas em 2013, a média de idade foi de 29 meses e 20 crianças eram do sexo masculino. O não aceite foi um dos motivos da perda de amostra, além do fato de algumas crianças estarem doentes no período da coleta (inverno no Rio Grande do Sul), ou pela dificuldade encontrada pelas mães em se deslocar ao laboratório.

O valor médio de hemoglobina foi de 11,8 g/dL. Apenas 8,3\% da amostra apresentou anemia, sendo a média de hemoglobina de 10,3 g/dL. A taxa de adesão à suplementação com sulfato ferroso foi de $80 \%$ no grupo avaliado em 2013. Contudo, o tempo médio de suplementação com sulfato ferroso foi de apenas 4,5 meses. Do total de crianças avaliadas, apenas 8 tiveram suplementação por tempo superior a 6 meses, sendo que estas apresentaram um aumento não significativo ( $\mathrm{p}=0,204)$ no valor de hemoglobina, conforme apresentado na Figura 1.

${ }^{11}$ O estudo foi aprovado pelo Comitê de Ética em Pesquisa da Universidade de Santa Cruz do Sul Universidade de Santa Cruz do Sul/UNISC (CAAE 06983012.0.0000.5343). 
Figura 1 - Nível sanguíneo de hemoglobina de acordo com o tempo de suplementação com sulfato ferroso em crianças matriculadas nas escolas de educação infantil do município de Venâncio Aires (RS) entre 2012 e 2013.

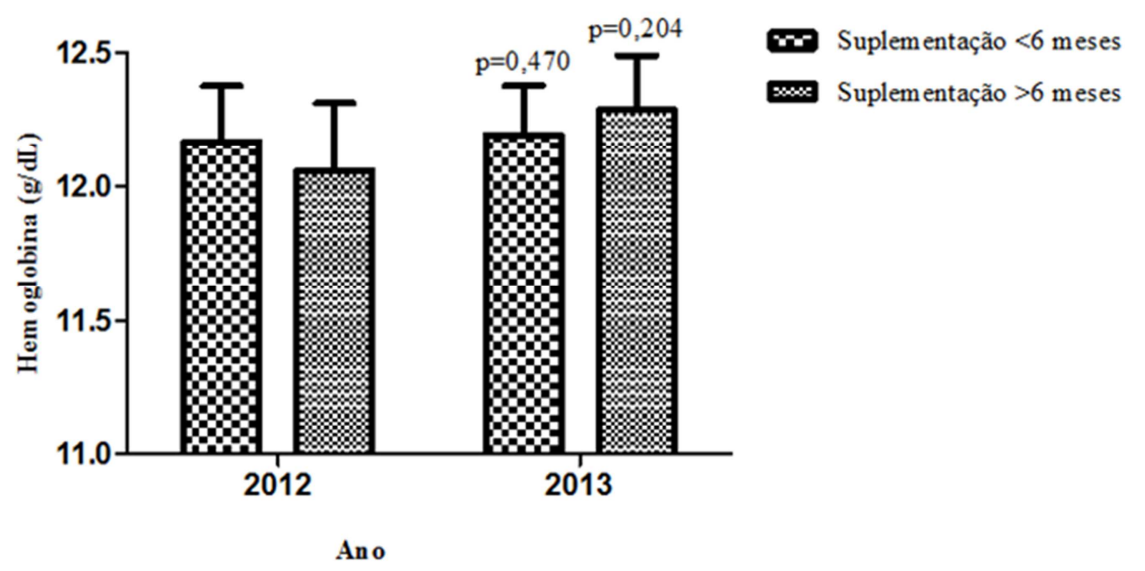

Participaram do estudo, em 2013, 36 crianças, pré-escolares matriculadas na rede pública do município de Venâncio Aires, RS, com idade entre 14 e 38 meses com o objetivo de reavaliar a presença de anemia, nas mesmas crianças que participaram do estudo em 2012, bem como avaliar a taxa de adesão e tempo de suplementação ao programa nacional de suplementação de sulfato ferroso das mesmas crianças avaliadas em 2012 e reavaliadas em 2013.

A presença de anemia encontrada na amostra de 2013 foi de 8,3\%, sendo considerada como um problema de saúde pública leve, de acordo com os critérios de classificação da OMS para prevalência de anemia. De acordo com a OMS, classifica-se em nível de importância como problema de saúde pública: grave, quando for encontrada a prevalência $\geq$ 40\%; nível moderado, quando a prevalência estiver entre 20,0 - 39,9\%; nível leve, quando a prevalência estiver entre $5-19,9 \%$, e como nível normal, no caso de prevalência $<4,9 \%$ (OMS, 2001).

Os resultados da Pesquisa Nacional de Demografia e Saúde (2006) mostraram que cerca de três milhões de crianças, com idade entre zero e 59 meses encontram-se anêmicas. Os maiores índices foram encontrados nas regiões Nordeste $(25,5 \%)$, Sudeste $(22,6 \%)$ e região Sul (21,5\%) (BRASIL, 2009). A prevalência da anemia no país vem aumentando consideravelmente nas últimas décadas, apontando para a urgente necessidade de medidas estratégicas de combate à anemia, como o incentivo ao aleitamento materno até os 6 meses, a alimentação complementar balanceada, o controle das parasitoses intestinais, a suplementação 
de ferro e a fortificação dos alimentos (SILVA et al., 2001; JORDÃO; BERNARDI; BARROS, 2009).

Jordão, Bernardi e Barros (2009) avaliaram fatores associados à prevalência da anemia na área rural do Nordeste Brasileiro, achando como resposta: baixo consumo de ferro heme pela população, devido às condições de pobreza e a falta de uma rede de distribuição de alimentos de origem animal; saneamento básico precário, desencadeando alto risco de parasitoses e condições desfavoráveis para o cultivo de frutas e verduras. Além desses, também estão relacionados à anemia outros fatores tais como a prematuridade, o baixo peso ao nascer, o abandono do aleitamento materno exclusivo precocemente e as famílias mais numerosas (KONSTANTYNE et al., 2007).

Além desses fatores encontrados nos estudos citados acima, destaca-se: a introdução precoce de leite de vaca, consumo excessivo de chás, baixa ingestão de vitamina $\mathrm{C}$, aleitamento materno prolongado (mais de 6 meses) sem suplementação de ferro, alimentação exclusiva com fórmulas infantis não fortificadas por mais de 4 meses, a não realização do prénatal e o baixo nível socioeconômico (NEUMAMM et al., 2000; SILVA et al., 2011).

Um estudo realizado com a mesma população em 2012 mostrou que 74\% das crianças consumiram papa salgada antes dos 6 meses de idade e 70,8\% consumiam chá. O baixo consumo de legumes e frutas foi relatado pelas mães quando questionadas sobre os hábitos alimentares das crianças, assim como o consumo regular de alimentos industrializados, ricos em açúcares e gorduras (PACHECO et al., 2013).

Dados da Pesquisa de Orçamentos Familiares (POF, 2008-2009) mostraram que frutas e verduras correspondiam a 2,8\% das calorias totais, quando o recomendado seria de 9 a $12 \%$ das calorias totais. Essa mesma pesquisa apontou que nos últimos 6 anos os alimentos básicos e tradicionais na alimentação brasileira (arroz, feijão e farinha de mandioca) perderam importância e a participação dos alimentos processados prontos para consumo aumentaram (pães, embutidos, biscoitos, refrigerantes e refeições prontas) (IBGE, 2010).

O tempo de suplementação, que deveria ser de pelo menos 6 meses, segundo a recomendação da OMS, ficou abaixo do recomendado (4,5 meses) e a adesão a suplementação na população estudada foi de 80 \%. Não existe parâmetro estabelecido para classificar adesão à suplementação.

A suplementação medicamentosa tem a vantagem de poder ser direcionada para a população de risco. Em 2005, o Ministério da Saúde (MS) criou o PNSF como uma estratégia para o controle da anemia no Brasil (BRASIL, 2005). Para o sucesso do PNSF é importante 
que as famílias se sensibilizem da importância da suplementação e da adesão a utilização do sulfato ferroso (BORTOLINI; VITOLO, 2007).

O estudo realizado em 2012 avaliou a prevalência de anemia, encontrando 11,5\% (PACHECO et al., 2013).Essa diminuição da anemia encontrada, que passou de 11,5\% para $8,3 \%$, pode estar relacionada à intervenção nutricional realizada no grupo em 2012, uma vez que as famílias foram orientadas sobre o consumo alimentar, alimentos fonte de ferro e maneiras de preparo dos alimentos.

Orientações para melhorar a disponibilidade de nutrientes dos alimentos, planejamento para garantir o acesso das famílias às fontes alimentares e estímulo para mudança nos hábitos alimentares são algumas ferramentas que podem contribuir para reverter a estimativa da anemia no país (ENGSTROM et al., 2008; ARCANJO et al., 2011; DURÁN, 2010). Mesmo havendo muitas causas para a deficiência de ferro, a alimentação costuma ser o principal fator contribuinte. A baixa ingestão de ácido fólico e vitaminas A, B12 e C também contribuem para o desenvolvimento da anemia (DURÁN, 2010; SAMMARTINO, 2010; KONSTANTYNER; TADDEI; PALMA, 2007; LOZOFF et al., 2006; OMS/UNICEF/UNU, 2001).

Observa-se em alguns casos que, no início da introdução à alimentação da criança, a oferta adequada de ferro não é suficiente para suprir sua necessidade. A falta de conhecimento das mães ou responsáveis sobre os alimentos fontes de ferro é um fator que contribui para essa realidade, assim como a dificuldade na aquisição desses alimentos devido às condições econômicas (SILVA et al., 2011). O início da ingestão insuficiente de ferro pela alimentação é um sinal de alerta muito importante, devido a possíveis chances de persistir essa oferta insuficiente por longo período, desencadeando uma anemia por deficiência de ferro (MOHAMED et al., 2010; SAMMARTINO, 2010).

Um fator que pode ter contribuído para a baixa presença de anemia encontrada no estudo, apesar do pouco tempo de suplementação, deve-se as orientações nutricional recebida após pesquisa de 2012 e à alimentação oferecida para essas crianças, uma vez que elas frequentavam a escola em turno integral e por isso recebiam uma alimentação balanceada, com oferta de alimentos fonte de ferro e alimentos fortificados. Nesse sentido, o fornecimento de cardápios equilibrados nas escolas são fundamentais para o fortalecimento da formação dos hábitos alimentares das crianças, na promoção e incentivo de uma alimentação variada, capaz de atingir as quantidades de micronutrientes necessárias a seu crescimento e desenvolvimento (THOMPSON, 2007). 


\section{CONCLUSÃO}

Foi observada adesão inadequada (tempo curto) à suplementação de sulfato ferroso, preconizado pelo programa nacional de suplementação de sulfato ferroso do Ministério da Saúde, e um baixo percentual de anemia $(8,3 \%)$, que pode se justificar pela orientação nutricional que o grupo recebeu após a pesquisa realizada em 2012.

A taxa de adesão ao programa nacional de suplementação de sulfato ferroso encontrada foi de $80 \%$. A adesão a esse programa de suplementação e aos demais programas implantados no Brasil é um verdadeiro desafio para a saúde pública, necessitando de novas estratégias. Outras variáveis relacionadas à anemia, como renda familiar, avaliação dos hábitos alimentares e consumo de alimentos fortificados devem ser avaliados em novos estudos.

A alimentação no início da vida é fundamental nesse processo. $\mathrm{O}$ aleitamento materno e a alimentação complementar saudável têm papel importante na prevenção da deficiência de ferro quando aliadas a outras medidas e essas práticas podem ser bem sucedidas por meio de ações efetivas dos profissionais de saúde. Orientações sobre hábitos alimentares saudáveis e modificação dietética podem levar à melhor seleção dos alimentos que contenham ferro e a um padrão de alimentação favorável para uma biodisponibilidade aumentada do ferro.

Novos estudos deverão ser desenvolvidos para avaliar os hábitos alimentares da população pré-escolar de Venância Aires, para confirmar a hipótese da alta qualidade nutricional da dieta do grupo.

\section{AGRADECIMENTOS}

Os autores agradecem à Universidade de Santa Cruz do Sul (UNISC) pela infraestrutura fornecida e à Fundação de Amparo à Pesquisa do Estado do Rio Grande do Sul (FAPERGS), à Coordenação de Aperfeiçoamento de Pessoal de Nível Superior (CAPES) e à UNISC pelas bolsas concedidas. 


\section{REFERÊNCIAS}

ARCANJO, F. P. N.; et al. Weekly iron supplementation for the prevention of anemia in preschool Children: A randomized, double-blind, placebo-controlled trial. Journal of Tropical Pediatrics, v. 57, n. 6, p. 433-438, 2011.

AZEREDO, C. M. et al. Efetividade superior do esquema diário de suplementação de ferro em lactentes. Revista de Saúde Pública, v. 44, n. 2, p. 230-239, 2010.

BRASIL. Ministério da Saúde. Secretaria de Atenção à Saúde. Departamento de Atenção Básica. Manual operacional do Programa Nacional de Suplementação de Ferro. Brasília: Ministério da Saúde, 2005.

BRASIL. Ministério da Saúde. Pesquisa Nacional de Demografia e Saúde da Criança e da Mulher - PNDS 2006: dimensões do processo reprodutivo e da saúde da criança. Brasília: Ministério da Saúde; 2009.

BORTOLINI, G. A.; VITOLO, M. R. Baixa adesão à suplementação de ferro entre lactentes usuários de serviço público de saúde. Pediatria (São Paulo), v. 29, n. 3, p. 176-82, 2007.

DURÁN, P. Anemia, problema complejo, soluciones simples? Archivos Argentinos de Pediatria, v. 108, n. 3, p. 197-200, 2010.

ENGSTROM, E. M. et al. Efetividade da suplementação diária ou semanal com ferro na prevenção da anemia em lactentes. Revista de Saúde Pública, v. 42, n. 5, p. 786-795, 2008.

JORDÃO, R. E.; BERNARDI, J. L. D.; BARROS, A. A. F. Prevalência da anemia ferropriva no Brasil: uma revisão sistemática. Revista Paulista de Pediatria, v. 27, n. 1, p. 90-98, 2009.

IBGE. Pesquisa de orçamentos familiares 2008-2009: despesas, rendimentos e condições de vida. Rio de Janeiro: IBGE, 2010.

KONSTANTYNER, T. et al. Fatores de risco de anemia em lactentes matriculados em creches públicas ou filantrópicas, São Paulo. Revista de Nutrição, v. 20, n. 4, p. 349-359, 2007.

LOZOFF, B. et al. Long-Lasting Neural and Behavioral Effects of Iron Deficiency in Infancy. Nutrition Reviews, v. 64, n. 5, p. S34-S43, 2006.

MOHAMED, M. W. et al. Methylphenidate improves cognitive deficits produced by infantile iron deficiency in rats. Behavioural Brain Research, v. 216, p. 146-152, 2010.

NEUMAN, N. A. et al. Prevalência e fatores de risco para anemia no Sul do Brasil. Revista de Saúde Pública, v. 34, n. 1, p. 56-63, 2000.

OMS/UNICEF/UNU. Iron deficiency anemia: assesssment, prevention, and control, a guide for programme managers. Genebra: WHO, 2001.

OMS. Worldwide prevalence of anemia 1993-2005. Genebra: WHO, 2008. 
PACHECO, J. P. et al. Prevalência da anemia em crianças de 6 a 24 meses matriculadas na rede pública de Venâncio Aires, RS, Brasil. Revista Jovens Pesquisadores, v. 3, p. 176-187, 2013.

PAIVA, A. A.; RONDÓA, P. H. H.; SHINOHARAB, E. M. G. Parâmetros para avaliação do estado nutricional de ferro. Revista de Saúde Pública, v. 34, n. 4, p. 421-426, 2000.

SAMMARTINO, G. Representaciones culturales acerca de la anemia y la suplementación conhierro. Archivos Argentinos de Pediatria, v.108, n. 5, p. 401-408, 2010.

SILVA, L. S. M. et al. Prevalência e determinantes de anemia em crianças de Porto Alegre, RS, Brasil. Revista de Saúde Pública, v. 35, n. 1, p. 66-73, 2001.

SILVA, E. B. et al. Fatores de risco associados a anemia ferropriva em crianças de 0 a 5 anos, em um município da região noroeste do Rio Grande do Sul. Revista Mineira de Enfermagem, v. 15, n. 2, p. 165-173, 2011.

THOMPSON, B. Food-based approaches for combating iron deficity. In: KRAEMER, K.; ZIMMERMANN, M. (Org.). Nutritional Anemia. Basel: Sight and Life Press, 2007. 